\title{
La integración de los procesos sustantivos universitarios desde el proceso extensionista: una mirada a la teoría
}

\section{The integration of university substantive processes from the extension process: a look at the theory}

\author{
MSc. Gisell Aguiar Melians ${ }^{1}$ \\ gaguiar@ucf.edu.cu \\ https://orcid.org/0000-0002-5387-5447 \\ Dra.C. Luisa María Baute Álvarez ${ }^{2}$ \\ Ibaute@ucf.edu.cu \\ https://orcid.org/0000-0001-9458-0795
}

Recibido: 1/4/2020; Aceptado: 1/6/2020

\begin{abstract}
RESUMEN
Este artículo tiene como objetivo sistematizar en el tratamiento a los procesos sustantivos universitarios con énfasis en la tercera función, la extensión universitaria, en el mundo y en Cuba, teniendo en cuenta la importancia de los mismos en la universidad actual para el perfeccionamiento de su misión, principalmente en la extensión universitaria como proceso sintetizador e integrador del quehacer universitario y su transmisión a la sociedad. A través del método histórico - lógico se realiza un análisis de diversas fuentes bibliográficas sobre la integración de los procesos universitarios y la labor extensionista, teniendo en cuenta las relaciones que se establecen entre ellos, así como de la participación protagónica universitaria, a partir de la formación de los futuros profesionales y la interacción de este en el entorno sociocultural y la comunidad. Se concluye con la importancia de la extensión universitaria para la interacción de la academia con la sociedad.
\end{abstract}

Palabras clave: extensión universitaria, proceso sustantivo, formación del profesional

\begin{abstract}
The aim of this article is to systematize in the treatment of the university substantive processes with emphasis on the third function, the university extension, in the world and in Cuba, taking into account the importance of these in the current university for the improvement of its mission, mainly in the university extension as a synthesizing and integrating process of university work and its transmission to society. Through the historical - logical method, an analysis of diverse bibliographical sources on the integration of university processes and extension work is carried out, taking into account the relationships established between them, as well as the participation of university protagonists, starting from the training of future professionals and their
\end{abstract}

\footnotetext{
${ }^{1}$ Universidad Carlos Rafael Rodríguez, Cienfuegos, Cuba.

2 Universidad Carlos Rafael Rodríguez, Cienfuegos, Cuba.
} 


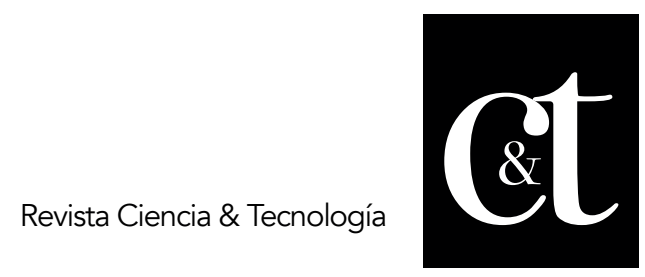

No. 27, 31 de julio de 2020

ISSN impreso: 1390 - 6321

ISSN online: 2661 - 6734

interaction in the sociocultural environment and the community. This research concludes with the importance of university extension programs with society.

Keywords: university extension, substantive process, professional training

\section{Introducción}

Las universidades han tenido históricamente la misión de preservar y promover la cultura acumulada por la sociedad a través de la formación personal, que se apropien de ella, la apliquen y para responder a demandas del desarrollo socioeconómico.

Las universidades cubanas buscan procesos de transformación para ofrecer respuestas a las demandas sociales; es necesario desarrollar gestión universitaria de excelencia, como expresara Miguel Díaz-Canel, entonces Ministro de Educación Superior en Cuba, en la conferencia de apertura del VII Congreso Internacional de Educación Superior Universidad 2010: "nuestra prioridad está dirigida a avanzar en el perfeccionamiento de la educación de las nuevas generaciones de profesionales cubanos, en la certeza de que la excelencia académica de la universidad cubana se mide a través de la comprobación de su pertinencia social" (Díaz Canel-Bermúdez, 2010).

Para cumplir este encargo, las IES cuentan con tres procesos esenciales que, ligados entre sí, tributan a su cumplimiento: los procesos de formación, investigación y extensión universitaria. Se ocupan de la generación, difusión y aplicación del conocimiento e implican un estrecho vínculo entre la institución y la sociedad, lo que incide en la formación del futuro egresado de la educación superior. La Universidad, en su papel de transformador del entorno, contribuye a que la sociedad gane características, una forma efectiva es la promoción de cultura artística, deportiva, la salud, así como de la ciencia, la tecnología y la producción y lo anterior remite a uno de los procesos sustantivos de la universidad: la Extensión Universitaria (EU).

Al decir de C. Tünnermann (2003), la extensión debe ser la mejor expresión de una integración creativa universidad-sociedad y su vínculo más idóneo; por lo que existe la posibilidad de hacer de esta función el eje de la acción universitaria, el hilo conductor de la inmersión social de la Universidad, con lo cual adquieren sus programas, una extraordinaria relevancia en el quehacer de las instituciones de educación superior para el mejoramiento de la calidad de su proceso formativo.

Teniendo en cuenta la importancia de este proceso sustantivo se aplicaron técnicas empíricas (entrevistas, observación y revisión documental) en la Facultad de Ciencias Económicas y Empresariales (FCEE) de la Universidad de Cienfuegos Carlos Rafael Rodríguez con el objetivo de conocer cómo se percibe la integración de los procesos sustantivos enfatizando en el proceso de extensión universitaria, revelándose las siguientes manifestaciones:

$\checkmark$ Falta de una concepción sistémica de la extensión universitaria como proceso.

$\checkmark$ A pesar de que la EU es uno de los procesos sustantivos conjuntamente con docencia e investigación sugestión se sigue orientando, fundamentalmente, a acciones que se relacionan con la cultura, el deporte y la promoción de salud.

$\checkmark$ Limitado alcance y participación de los actores de la comunidad intra y extra universitaria por la estrecha acepción de cultura que hoy persiste. 


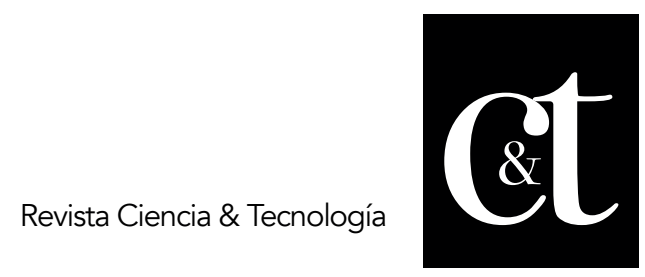

No. 27, 31 de julio de 2020

ISSN impreso: 1390 - 6321

ISSN online: 2661 - 6734

$\checkmark$ Se realizan acciones propias de la extensión universitaria en los procesos de docencia e investigación que, por lo general no se consideran como tal.

$\checkmark$ Profesores y otros integrantes de la comunidad universitaria presentan limitaciones para gestionar la EU debido al desconocimiento de los referentes teóricos - metodológicos para su planeación, puesta en marcha y evaluación.

$\checkmark$ La insuficiente orientación en los documentos esenciales de las carreras, (planes de estudio, programas de asignaturas.

$\checkmark$ Ambigüedad con que se ha manejado el concepto de EU

La situación anterior reveló el siguiente problema científico: es insuficiente el conocimiento de la integración de los procesos universitarios con énfasis en la EU en la comunidad intra universitaria de la FCEE. Es por ello que este estudio propone sistematizar en el tratamiento a la integración de los procesos sustantivos universitarios con énfasis en la tercera función para contribuir a la elevación del nivel de conocimientos de los diferentes actores de la FCEE en este sentido, para así perfeccionar la gestión de la EU.

\section{Materiales y Métodos}

La realización de este estudio estuvo sustentada en la revisión bibliográfica, pues se planteó profundizar en los múltiples enfoques sobre la extensión universitaria, los procesos sustantivos y su integración en las instituciones de Educación Superior, que han sido estudiados desde diversas perspectivas en contextos diferentes, empleando el método histórico-lógico que posibilitó el análisis de la evolución en el contexto internacional de la temática. Se empleó el método inductivo-deductivo con el cual se pudo llegar a conclusiones en relación con las concepciones existentes en la bibliografía sobre la integración de los procesos sustantivos universitarios, enfatizando en la extensión universitaria.

Se trabajó con métodos del nivel empírico como la observación, que permitió recuperar información acerca de características de los procesos sustantivos; así como el análisis de documentos sobre procesos y entrevistas a profesores y directivos académicos para conocer el nivel de conocimiento de la integración de los procesos universitarios con énfasis en la EU en la comunidad intra- universitaria de la FCEE.

\section{Desarrollo}

En el prefacio del comunicado de la Conferencia Mundial sobre la Educación Superior - 2009, se hace referencia a la responsabilidad social que tiene la educación superior de hacer avanzar en la comprensión de problemas polifacéticos con dimensiones sociales, económicas, científicas y culturales, así como en la capacidad de hacerles frente, y la importancia de su asunción en el liderazgo social en materia de creación de conocimientos de alcance mundial para abordar retos mundiales. Por ello las IES deben lograr la armonía en el funcionamiento de sus procesos fundamentales para que, de una manera sinérgica, puedan contribuir al cumplimiento de la misión social que, como institución educativa, desempeñan.

La necesidad de "perfeccionar y dinamizar el modelo de gestión de las universidades..." (Ortiz, 2014) quedó reafirmada en el Congreso Internacional de Educación Superior Universidad 2014, en la conferencia inaugural del Ministro de Educación Superior en Cuba, Rodolfo Alarcón Ortiz, cuando asevera que la responsabilidad social universitaria requiere "cambios cualitativos en nuestro modelo de gestión para garantizar la integración de los procesos sustantivos" (Ortiz, 2014). 


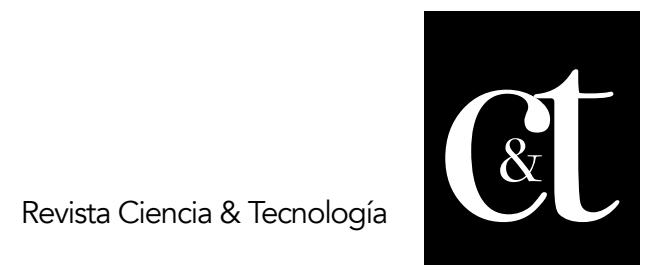

No. 27, 31 de julio de 2020

ISSN impreso: 1390 - 6321

ISSN online: 2661 - 6734

Por ello, asumir una gestión responsable pudiera contribuir a erradicar algunas deficiencias que se presentan en este sentido, lo que implicaría la realización de cambios en las políticas de gestión, que se sustentan, entre otros aspectos, en nuevas estrategias de planificación y de evaluación participativas. Lo anterior no se aparta de las concepciones teóricas más actuales sobre la responsabilidad social que tiene la Universidad, en su rol de transformadora de su entorno, pues esta resulta una vía para contribuir a que las IES eleven la calidad de los tres procesos sustantivos en la Universidad, (docencia, investigación y extensión universitaria). De igual manera, se puede garantizar una gestión más eficiente de los impactos para hacer cumplir la misión de las IES en la sociedad, comprometiendo a los actores sociales con su quehacer, en tanto les impone retos como ciudadanos responsables y protagonistas del medio donde se desempeñan.

Esta es una razón importante para que la educación superior profundice en el estudio de sus componentes, interrelaciones y procesos que desarrolla y que le aportan significación integral y oportunidades para la transformación social. Debe priorizar el perfeccionamiento constante de sus procesos sustantivos: docencia, investigación y extensión, con el objetivo de cumplir su misión de favorecer una actitud de cambio a través de los profesionales que egresan de las IES, a base de formación cada vez más integral y una conciencia ética (González y González, 2002; Galarza, 2007).

Estos procesos sustantivos, (también denominados como claves o principales), son aquellos que inciden de manera significativa en los objetivos estratégicos y son críticos para el éxito de la organización, además son los que aportan valor. Por esta razón, resulta de vital importancia, en estas organizaciones, poder desarrollar, cada vez con más intencionalidad, la gestión de sus procesos sustantivos, mediante la planificación, organización, ejecución y control (Álvarez, 2002; González y González, 2002; Horruitiner, 2006; Ortiz, 2014), para obtener resultados superiores con el objetivo de lograr la excelencia y la satisfacción de la sociedad.

En este sentido, los tres procesos, la docencia como proceso de enseñanza y aprendizaje de los conocimientos que se producen a través de la investigación científica representada en las diferentes ciencias y que constituye el contenido de las disciplinas, deben llegar al entorno social para la satisfacción de las necesidades de desarrollo cultural y la solución de problemas de la práctica social, siendo entonces la extensión universitaria quien los interrelaciona a través de la promoción de estas acciones. La labor extensionista que se lleva a cabo en la universidad se realiza como un proceso aislado, lo que limita su integración con los dos restantes procesos universitarios. Aun realizando acciones en procesos de formación e investigación que pueden considerarse como acciones de extensión no se tienen en cuenta como tal.

Con respecto a lo anterior, la práctica de la EU debe direccionarse desde la clase, explotando las potenciales que esta puede aportar para desarrollarla. Sin embargo, en la práctica laboral se evidencia más esa interacción con la realidad circundante a la que se refieren Galarza (2007) y Almuiñas, Del Huerto y Caranes (2001), pues se aplican los conocimientos adquiridos en el contexto laboral que, indudablemente, resulta muy necesario para la formación del profesional, especialmente del economista, por la importancia que su labor tiene en el perfeccionamiento del modelo económico y la planificación de la economía en nuestro país.

Es por ello que la formación del futuro profesional no debe quedar ajena al entorno, que es donde desarrollará su actividad profesional, donde será capaz de satisfacer 


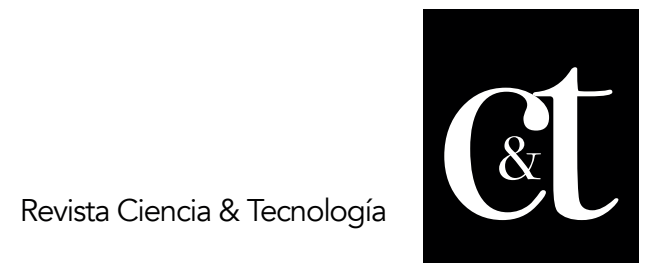

No. 27, 31 de julio de 2020

ISSN impreso: 1390 - 6321

ISSN online: 2661 - 6734

sus necesidades individuales y sociales, al tiempo que debe estar a tono con el desarrollo de las fuerzas productivas lo que requiere la acción de la ciencia y la técnica, siendo este el objetivo principal en su formación. Desde la aparición de la extensión en la escena universitaria, se consideró como una función institucional, con cierta relevancia, a través de la cual es posible llevar a cabo la retroalimentación de tareas académicas de la institución. Ella requiere expresar los máximos valores que desarrolla la institución y ser asumida con responsabilidad plena por parte de todos sus actores.

El concepto de extensión universitaria aparece en 1898, introducido por el español Adolfo González, quien la declara como toda labor expansiva de carácter educativo y social, realizada por la universidad fuera de su esfera oficial docente, siendo las actividades de difusión cultural y de divulgación científica dirigidas a sectores populares de la sociedad.

El fortalecimiento de la función social de la universidad, con la proyección de su quehacer a la sociedad mediante los programas de extensión universitaria y difusión cultural, estuvo presente desde muy temprano entre los postulados de la Reforma de 1918; siendo entonces, la "misión social" de la universidad un remate programático de la Reforma. Así, el movimiento agregó al tríptico misional clásico de la universidad, un nuevo y prometedor cometido, capaz de vincularla más estrechamente con la sociedad y sus problemas, de volcarla hacia su pueblo, haciendo a este partícipe de su mensaje y transformándose en su conciencia cívica y social. A partir de este momento, se concibió toda una gama de actividades que generó el ejercicio de esta misión social, que incluso se tradujo en determinados momentos en una mayor concientización y politización de los cuadros universitarios, contribuyendo a definir el perfil de la universidad latinoamericana, al asumir ésta, o sus elementos componentes, tareas que no se proponían o que permanecían inéditas para las universidades de otras regiones del mundo.

En la I Conferencia Latinoamericana de Extensión Universitaria y Difusión Cultural, en Santiago, y aprobó recomendaciones que precisan la teoría latinoamericana sobre esta materia, se adopta el siguiente concepto de extensión (Azócar, 2012):

La extensión universitaria debe ser conceptuada por su naturaleza, contenido, procedimientos y finalidades, de la siguiente manera: por su naturaleza, la extensión universitaria es misión y función orientadora de la universidad contemporánea, entendida como ejercicio de la vocación universitaria. Por su contenido y procedimiento, la extensión universitaria se funda en estudios y actividades filosóficas, científicas, artísticas y técnicas que exploran y recogen del medio social, nacional y universal, los problemas, datos y valores culturales que existen en todos los grupos sociales. Por sus finalidades, debe proponerse, como fines fundamentales proyectar dinámica y coordinadamente la cultura y vincular a todo el pueblo con la universidad. Además de dichos fines, la extensión universitaria debe procurar estimular el desarrollo social, elevar el nivel espiritual, intelectual y técnico de la nación, proponiendo, imparcial y objetivamente ante la opinión pública, las soluciones fundamentales a los problemas de interés general. Así entendida, la extensión universitaria tiene por misión proyectar, en la forma más amplia posible y en todas las esferas de la nación, los conocimientos, estudios e investigaciones de la universidad, para permitir a todos participar en la cultura universitaria, contribuir al desarrollo social y a la elevación del nivel espiritual, moral, intelectual y técnico (pp.1) 


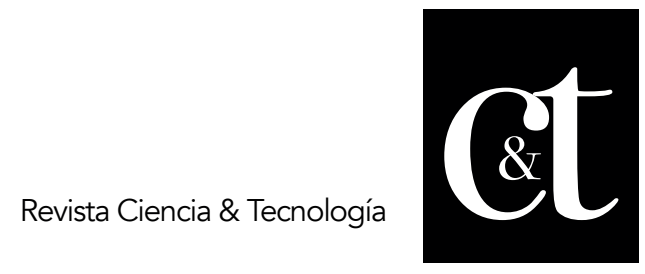

No. 27, 31 de julio de 2020

ISSN impreso: 1390 - 6321

ISSN online: 2661 - 6734

En este concepto de extensión universitaria y difusión cultural, predominante por varias décadas, se caracterizó por incorporar las tareas de extensión y difusión al quehacer normal de la universidad, pero se consideraban como una proyección a la comunidad de ese quehacer, como una extensión de su radio de acción. Puede decirse que, existió un predominio de "entregar" con un marcado acento "paternalista" o "asistencial" en las labores que se realizaban. La universidad, sin embargo, a sabiendas de su condición de institución superior del saber, trataba de remediar esta situación privilegiada y procuraba que algo de su quehacer se proyectara a los sectores menos favorecidos. Esa proyección, en que la universidad es la que da y la colectividad la que recibe, establecía un canal de una sola vía que va de la universidad, depositaria del saber y la cultura al pueblo, simple destinatario de esa proyección y al cual se supone incapaz de aportar nada valioso.

La extensión ha ido evolucionando y se ha consolidado como función y proceso inherente a la universidad; se va perfeccionando el concepto, en el que varios autores han realizado aportes. Entre ellos Guédez y otros (1988), que en estudios realizados declaran que la extensión universitaria es el espacio donde debe prevalecer el sentido comunicativo entre la universidad y su entorno, según el propósito de promover el ser de la comunidad a través del quehacer de la universidad, así como fomentar el quehacer de la comunidad mediante la proyección del ser de la universidad.

En el Programa Nacional de Desarrollo de la Extensión Universitaria en 1988, el concepto de extensión se expresa como el "conjunto de acciones que realiza el centro, dentro y fuera de sus instalaciones, dirigidas a los estudiantes y trabajadores, y a la población en general, con el propósito de promover y difundir la cultura en su más amplia acepción, es decir, la cultura científica, la técnica, la política, la patrióticomilitar e internacionalista, la artística y literaria, la física, etc. González y González, (2002) conceptualizan la extensión universitaria como sistema de interacción de la universidad y la sociedad, a través de actividad y comunicación, realizado dentro y fuera de la institución de educación superior para promover la cultura en la comunidad universitaria y extrauniversitaria y contribuir a su desarrollo en la cultura. En las anteriores posiciones se refirma la extensión universitaria como fortalecedora de la promoción de cultura entre la sociedad y la universidad. Se infiere que se limita a la intención de pronunciarse desde los objetivos de la universidad.

Fernández-Larrea, conjuntamente con González, (1999-2000) llegan a una concepción más esencial de extensión universitaria al definirla como: "el proceso que tiene como propósito promover la cultura en la comunidad intrauniversitaria y extrauniversitaria, para contribuir a su desarrollo cultural." (González y Fdez-Larrea, 2001) Esta concepción resulta muy interesante pues, direccionan la labor extensionista desde la comunidad intrauniversitaria como parte de la extrauniversitaria, lo que permite la comprensión de que la promoción cultural se realiza dentro de la casa de altos estudios y fuera de esta, potenciando el desarrollo cultural de la comunidad universitaria y la comunidad social.

Vega Mederos (2002) se refiere a que para que la universidad cumpla con su encargo social tiene la necesidad de desarrollar un sistema de interacciones recíprocas que englobe a las misiones de la universidad, entiéndase docencia, investigación y extensión pues, ellas parten y se nutren del referente social y sus resultados se revierten en ellas, afirmando que este componente se convierte, a partir de su práctica, en el elemento dinamizador que facilita el flujo continuo de conocimientos compartidos entre la universidad y la sociedad, que los enriquecen mutuamente. 


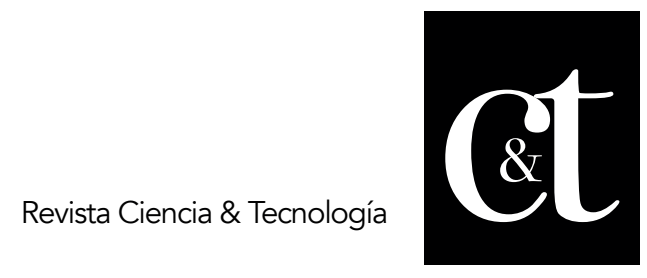

No. 27, 31 de julio de 2020

ISSN impreso: 1390 - 6321

ISSN online: 2661 - 6734

Tünnerman (2003) plantea que consecuente con el criterio de que la extensión debería ser la mejor expresión de una integración creativa universidad-sociedad y su vínculo más idóneo, existe la posibilidad de hacer de esta función el eje de la acción universitaria, el hilo conductor de la inmersión social de la universidad, con lo cual adquieren sus programas una extraordinaria relevancia en el quehacer de las instituciones de educación superior, de cara al nuevo milenio. La extensión, acorde con la naturaleza del conocimiento contemporáneo, debe estructurarse sobre la base de equipos interdisciplinarios. Además, si la extensión es la función universitaria más próxima a la realidad social, por principio tiene que ejercerse interdisciplinariamente, desde luego que esta es la única manera de acercarse a la realidad, que por naturaleza es interdisciplinaria. Los sectores a los que van dirigidos los programas de extensión adquieren aprendizajes que les permitan dar continuidad a los proyectos. Los diseños programáticos de la extensión deben dar espacio a la autocrítica y prever mecanismos de evaluación permanente para la retroalimentación de los proyectos.

Las apreciaciones de los autores antes mencionados propician la reflexión sobre la importancia de que los tres procesos fundamentales de la universidad, deben caminar juntos pues, uno depende de otro para su buen desarrollo. No puede verse separada la formación de la investigación porque a partir de la primera se aportan conocimientos para que la segunda se realice y desarrolle. La segunda consolida y aplica los conocimientos adquiridos en la docencia y, por supuesto, la extensión permite sistematizarlos a los dos, como una vía efectiva para su socialización. Desde la perspectiva de Del Huerto (2001) se precisa la extensión como proceso formativo, integrador y sistémico, basado en las interacciones culturales del quehacer universitario en comunicación bidireccional con la sociedad, incluyendo a la comunidad universitaria, orientado a la transformación social.

Se refiere a la bidireccionalidad de la comunicación, es decir, a que no solo es la trasmisión e información del quehacer universitario, sino el necesario contacto con la sociedad para poder responder a sus necesidades concretas, sin embargo, puede decirse que no solo deben tenerse en cuenta en esta comunicación las necesidades sociales, sino también sus logros y perspectivas como actores participantes en el proceso extensionista.

Fernández-Larrea (2002) considera que el proceso extensionista es resultado de las relaciones sociales que se dan entre los sujetos que en él participan está dirigido de un modo sistémico y eficiente, a la promoción de la cultura para la comunidad intra y extrauniversitaria, con vistas a la solución del problema social a través de la participación activa de la comunidad universitaria y extrauniversitaria, planificada en el tiempo y observando ciertas estructuras organizativas, con ayuda de objetos, instrumentando indicadores que midan calidad y cuyo movimiento está determinado por relaciones causales entre sus componentes y de ellos con la sociedad.

Si se compara esta concepción con la anterior aportada por esta misma autora entre los años 1999 y 2000, puede apreciarse un cierto grado de madurez pues, identifica a la extensión universitaria como un proceso, donde se integran sus contenidos, refiriendo la necesidad de incorporar métodos, medios y recursos para su materialización. Asimismo, se evidencia la importancia de la evaluación para su perfeccionamiento; aspectos que, hasta ese momento, constituían vacíos en los presupuestos anteriores, siendo necesarios estos para alcanzar un mayor y mejor desarrollo de la extensión universitaria. Sin embargo, teniendo en cuenta la opinión 


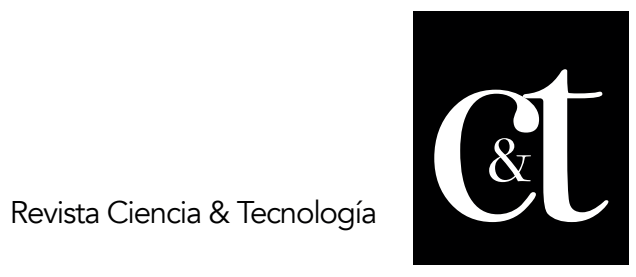

No. 27, 31 de julio de 2020

ISSN impreso: 1390 - 6321

ISSN online: 2661 - 6734

de Medina, (2011) se debe destacar que se considera una limitación que la participación activa de la comunidad universitaria y extrauniversitaria se visualice como método, cuando por la importancia que tiene, debiera verse como objetivo de la extensión universitaria. Además, las autoras del presente trabajo, consideran que se hace necesario, cuando se habla de promoción de la cultura acumulada, no solo se refiere a la cultura deportiva, artística, literaria y de salud, como es habitual en la labor extensionista, sino también a la adquirida desde los conocimientos propios de la profesión.

En el Programa Nacional de Extensión Universitaria aprobado en el año 2004, el concepto de extensión es enunciado como "proceso que tiene como objetivo promover la cultura en la comunidad intrauniversitaria y extrauniversitaria, para contribuir a su desarrollo cultural". Se reafirma el carácter de proceso, sin embargo, no se aprecia la figura del estudiante como centro del mismo, siendo estos el eslabón principal en la promoción de la cultura que produce y preserva la universidad y, por tanto, una fuerza importante para la transformación de la sociedad.

Según Pérez de Maza (2011), la extensión emerge como función que procura la relación dialéctica universidad-sociedad, mediante su integración con la docencia y la investigación. Plantea que en esta relación la IES obtiene retroalimentación del servicio público que presta, de la pertinencia del currículum y de sus prácticas educativas e institucionales, al establecer retos que demandan el cambio y la transformación de la universidad para atender los requerimientos de la sociedad. La anterior concepción, resulta muy abarcadora, pues en ella se recoge la esencia misma de la extensión universitaria al hacer referencia a la relación entre la universidad y la sociedad, la integración entre los tres procesos sustantivos de la universidad.

Teniendo en cuenta lo anterior pudo llegarse a que la extensión universitaria es un proceso que, por excelencia, vincula e inserta a la universidad con la sociedad, integrando los tres procesos sustantivos universitarios: formación, investigación y la propia extensión universitaria, sin desestimar las características y necesidades de las comunidades en las que se inserta, incidiendo en su desarrollo socioeconómico, retroalimentándose del mismo, toda vez que conoce y evalúa el impacto de sus acciones en el entorno y, a su vez, perfeccionar la formación de los futuros egresados.

\section{Resultados}

Las IES preservan, desarrollan y promueven la cultura acumulada por la sociedad y por tanto deben encargarse de la formación, trasmisión y apropiación de valores y conocimientos, de desarrollo de habilidades, actitudes, destrezas, así como de la formación de capacidades, garantes de los valores universales y del patrimonio cultural para contribuir a la transformación del entorno inmediato, natural, social y cultural, enfatizando en la idea de la estrecha relación que tiene que existir, necesariamente, entre sus funciones sustantivas para cumplir su encargo social.

Para ello aglutinan su quehacer y proyectarlo, a través de la Extensión Universitaria, facilitando el vínculo universidad-sociedad a través de la promoción de las diferentes formas de cultura (científico-tecnológica, artístico-literaria, cultura física, de salud, socio-humanística, económica, jurídica, político-ideológica, entre otras), para dar respuesta a las necesidades de superación y capacitación, por lo que contribuye al desarrollo cultural integral. Es así que la EU tiene la cualidad de ser integradora, pues se nutre de los contenidos de los demás procesos, aunque tiene personalidad propia y totalizadora. Esta cualidad de la EU sustenta un nuevo paradigma para este 


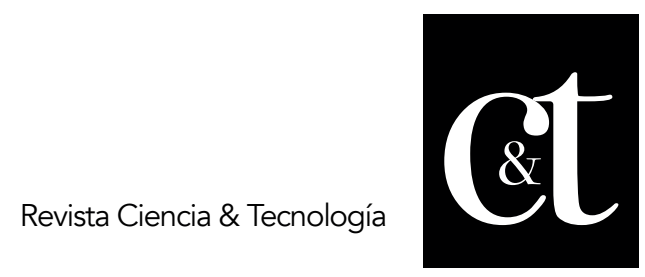

No. 27, 31 de julio de 2020

ISSN impreso: 1390 - 6321

ISSN online: 2661 - 6734

proceso-función que descansa en un concepto amplio, diversificado con su consecuente aplicación en la práctica, asumiéndolo como un proceso formativo, dinámico, integrador y multifacético (Batista, Barbán y Trujillo, 2016).

En este sentido, resulta evidente que, el eslabón fundamental en la estructura de las universidades actuales, es el departamento docente pues donde se integran una o varias disciplinas $y$, a su vez, en él se concretan las funciones sustantivas universitarias. Por esta razón, el departamento docente no puede limitarse únicamente a la actividad académica en la que el estudiante se apropia de la cultura de la humanidad.

La tendencia no puede ser que en la universidad sea el espacio donde se formen profesionales o especialidades en el que predomine lo académico y que la actividad científica sea únicamente para los centros o laboratorios de investigación pues esto conduciría a la creación de una mentalidad dirigida a la docencia, con poco interés por la investigación y, como consecuencia, quedarían separadas dichas actividades, lo que trae consigo un muy limitado desarrollo de proyectos en todos los sentidos $y$, por tanto, un estancamiento palpable del conocimiento y de las innovaciones que se podrían llevar a cabo en pos del mejoramiento económico, cultural y social. Además, como se ha planteado anteriormente el conocimiento se aplica y consolida en las investigaciones y la mejor manera de desarrollarlas es dándolas a conocer, socializarlas y es aquí donde la extensión juega su papel más importante: insertar la Universidad en la sociedad.

Esta inserción no debe limitarse solamente a la promoción de acciones en el entorno social, para satisfacer las necesidades del desarrollo cultural, sino que debe conocer sus necesidades, problemas y capacidades para desarrollar investigaciones, proyectos e innovaciones que permitan un mayor y mejor desarrollo de la sociedad. Puede decirse entonces que la extensión implica un aprendizaje recíproco, pues al conocer la IES, la realidad nacional, puede enriquecer y redimensionar toda su actividad académica.

El espacio educativo, por el alcance y repercusión que tiene tanto en estudiantes como en sus familias y comunidades es ideal para realizar acciones que promuevan el conocimiento de la profesión y de índole general, que aporten al crecimiento individual y social y así lograr bienestar en los estudiantes y en su calidad de vida, por lo que en la propia formación de los profesionales debe tenerse en cuenta los patrones culturales desde la óptica de las profesiones. La producción científicotecnológica se inscribe como proceso continuo de consolidación de la cultura humana y la extensión es la que se encarga de promover la cultura entre la universidad y su entorno social interno y externo. Partiendo de esta afirmación es que se plantea que la extensión universitaria es una función sustantiva que ocupa un lugar especial, por su profundo contenido cultural, en el sentido más amplio de la palabra, que transversaliza los procesos de docencia y de investigación y que, además, participa como proceso en el sistema de relaciones entre la institución formativa y la sociedad.

Sin embargo, la extensión universitaria, sigue presentando dificultades con su misión pues, muchas universidades insisten en ser generadoras de conocimientos y su difusión con el fin de lograr el mayor impacto posible en el bienestar social, y esto implica que las investigaciones se desarrollen a partir de las temáticas que trabajan los investigadores y por tanto no se socializan totalmente, sino que existe cierta tendencia a apropiarse de los resultados. Además, persiste la práctica de asilar la 


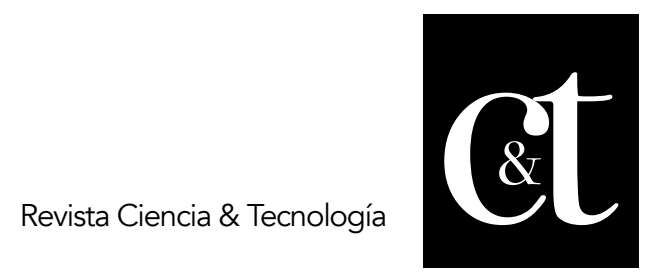

No. 27, 31 de julio de 2020

ISSN impreso: 1390 - 6321

ISSN online: 2661 - 6734

extensión universitaria en su arista artística y de actividades de corte político y recreativo sin tomar en cuenta que es la vía idónea para poner en conocimiento de toda la sociedad, no solo los aprendizajes sino también de las investigaciones, proyectos e innovaciones realizadas en función de resolver problemas de la misma.

\section{Conclusiones}

Las IES como generadoras y difusoras del conocimiento acumulado por la sociedad, inevitablemente deben integrar los procesos sustantivos universitarios para, de esta manera, dotar a los futuros egresados de las herramientas necesarias para su desarrollo profesional. Asimismo, resulta de gran importancia, la interacción directa con la sociedad para conocer sus necesidades, problemáticas principales, habilidades y capacidades para lograr transformarla es aras del bienestar económico, cultural y social; siendo la extensión universitaria la vía más efectiva para lograr esta interacción.

\section{Referencias bibliográficas}

Alarcón Ortiz, Rodolfo. (2014). Conferencia inaugural dictada en el X Congreso Internacional de Educación Superior Universidad 2014 (pp. 1-8). Presentado a Universidad innovadora por un desarrollo humano sostenible: Mirando al 2030, La Habana. Recuperado de https://transformacion.reduc.edu.cu/index.php/transformacion/article/view/243 $\angle 174$

Almuiñas, J. L. Del Huerto, M. y Caranes, J. L. (2001). La extensión universitaria: Un reto a la gestión cultural de las universidades del Siglo XXI. Madrid: Trabe S. L.

Azócar, E. (2012). Apuntes históricos de la extensión universitaria. Descargado de: https://www.aporrea.org/educacion/a155057.html

Batista, D., Barbán, Y., y Trujillo, Y. (2016). El método participativo integrador extensionista y sus procedimientos para la formación integral del estudiante. Revista Magazine de las Ciencias. Vol. 1 Número 3; pp. 49-66.

Del Huerto, M. (2007). La extensión universitaria como vía para fortalecer los vínculos universidad-sociedad desde la promoción de la salud. Revista cubana de salud pública. V. 33 (2);

Díaz-Canel Bermúdez, M. (2010). Conferencia Inaugural Congreso Internacional de la Educación Superior. (p. 24). Presentado a La Universidad ante los retos de la construcción de la sociedad socialista en Cuba., La Habana, Cuba. Recuperado de http://www.congresouniversidad.cu

Fernández Larrea, G. (2002). Modelo de gestión de la extensión universitaria para la Universidad de Pinar del Río (Tesis en opción al grado científico de Dr. en Ciencias de la Educación), Universidad de Pinar del Río Hermanos Saíz Montes de Oca, Pinar del Río, Cuba.

Galarza, J. (2007, enero-abril). La Evaluación de la gestión de los procesos estratégicos en las Instituciones de Educación Superior: una perspectiva para el desarrollo de la gestión institucional. Revista Cubana de Educación Superior, XXVII (1), 106-116.

González, G. R. y González Fernández, M. (2002). La Gestión de la Extensión Universitaria: una aproximación desde la perspectiva cubana. Revista Cubana de Educación Superior, XXII (2), 55-68. 


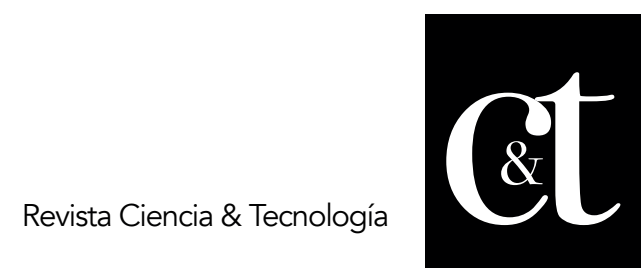

No. 27, 31 de julio de 2020

ISSN impreso: 1390 - 6321

ISSN online: 2661 - 6734

Horruitiner Silva, P. (2006). La Universidad Cubana: el modelo de formación. La Habana: Cuba. Ed. Félix Varela.

Medina Yera, E. (2011). Modelo pedagógico de formación del protagonismo del futuro profesional desde la extensión universitaria, en el contexto de la universalización. Universidad de Ciencias Pedagógicas "Frank País García," Santiago de Cuba.

Pérez de Maza, T. (2011). Extensión universitaria: función organizadora de un currículum abierto. Universidad Nacional de Educación a Distancia, Madrid, España.

Programa Nacional de Desarrollo de la Extensión Universitaria (1988). Ministerio de Educación de la República de Cuba. La Habana. Recuperado de https://www.mes.gob.cu/sites/default/files/documentos/EU_PNEU.pdf

Suárez Ramírez, A. (2017). La preparación de los profesionales de la educación en formación inicial para el desarrollo cultural educativo de la comunidad extrauniversitaria. Universidad Central Marta Abreu de las villas, Santa Clara.

Tünnermann, C. (2000). El nuevo concepto de la extensión universitaria y difusión cultural y su relación con las políticas de desarrollo cultural en América Latina. Anuario de Estudios Centroamericanos, 4: 93-126.

Vega Mederos, J. (2002). La extensión universitaria: función integradora del quehacer universitario en el presente siglo. Investigación y Desarrollo, 1, 10, 2639. 